\title{
Proton-Beam-Driven Plasma Acceleration
}

\author{
Erik Adli \\ Department of Physics, University of Oslo, N-0316 Oslo, Norway \\ erik.adli@fys.uio.no \\ Patric Muggli \\ Max Planck Institute for Physics, Munich, Germany \\ muggli@mpp.mpg.de
}

\begin{abstract}
We describe the main ideas, promises and challenges related to proton-driven plasma wakefield acceleration. Existing high-energy proton beams have the potential to accelerate electron beams to the $\mathrm{TeV}$ scale in a single plasma stage. In order to drive a wake effectively the available beams must be either highly compressed or microbunched. The self-modulation instability has been suggested as a way to microbunch the proton beams. The AWAKE project at CERN is currently the only planned proton-driven plasma acceleration experiment. A self-modulated CERN SPS beam will be used to drive a plasma wake. We describe the design choices and experimental setup for AWAKE, and discuss briefly the short-term objectives as well as longer-term ideas for the project.
\end{abstract}

Keywords: Plasma wakefield acceleration; proton beams; CERN; AWAKE.

\section{Introduction}

A plasma wakefield accelerator is an energy transformer. A drive beam propagates through a plasma, creating a strong plasma wake and in the process losing energy to the plasma. Part of this energy can be used to accelerate a trailing beam, here called the "witness beam." The driver can be a highintensity laser beam, where the ponderomotive force of the laser pulse drives the plasma wake $[1,2]$, or a charged particle beam, where the space charge force drives the plasma wake [3, 4]. For laser drivers, the technique is called "laser wakefield acceleration" (LWFA), while for particle beam drivers, the term "plasma wakefield acceleration" (PWFA) has been adopted. For both techniques, the amount of energy available for acceleration is given by the energy of the driver and the energy transfer efficiency from the drive beam to the witness beam. In order to efficiently drive the plasma with a particle bunch, the driver length $\sigma_{z}$ should be such that $k_{\mathrm{pe}} \sigma_{z} \simeq \sqrt{2}[5]$, where $k_{\text {pe }}=2 \pi / \lambda_{\text {pe }}$, with $\lambda_{\text {pe }}$ being the cold electron plasma wavelength. The transverse spot size $\sigma_{r}$ should be $k_{\mathrm{pe}} \sigma_{r} \lesssim 1$ in order to avoid development of filamentation instability [6]. Figure 1(a) illustrates a drive-witness bunch configuration.
Assuming dephasing or emittance-driven head erosion [7] are not limiting factors, the drive beam can drive the wake until its energy has been depleted. At this point a new drive beam must be injected into a new plasma stage in order to accelerate the witness beam to higher energies, as illustrated in Fig. 1(b). Injection and ejection of the drive beam requires a potentially long and complex interstage beam line between the plasma stages [8], resulting in a reduction of the average accelerating gradient. The energy gain of a witness particle in a single plasma stage may also be limited by the transformer ratio, defined as the ratio of the accelerating field within the witness bunch to the decelerating field within the drive bunch. For a symmetric bunch and for linear wakes, the transformer ratio is limited to 2 , and the witness particle energy gain limited to twice the drive particle energy. Simulations studies indicate that bunch shaping [4, 9] or bunch trains $[4,10]$ may provide larger transformer ratios. However, transformer ratios of 2 or more have so far not been demonstrated experimentally in PWFA two-bunch experiments [11], and hightransformer-ratio PWFA remains a field of active research. The energy gain of a witness bunch in a single stage may therefore be limited by either the drive particle energy or the drive beam total energy. 


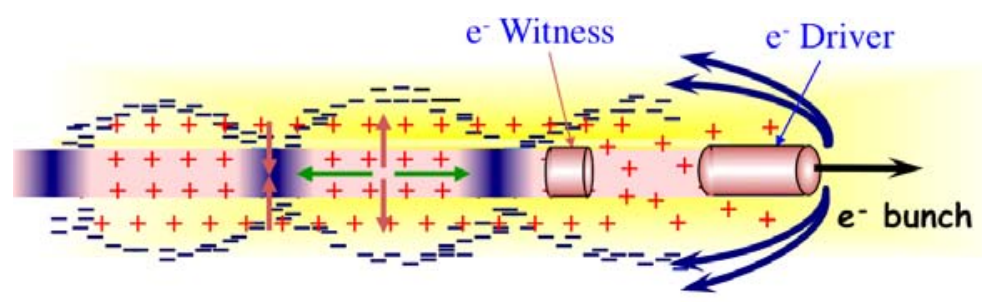

(a)

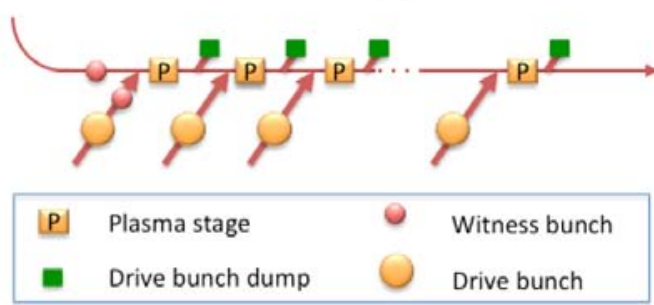

(b)

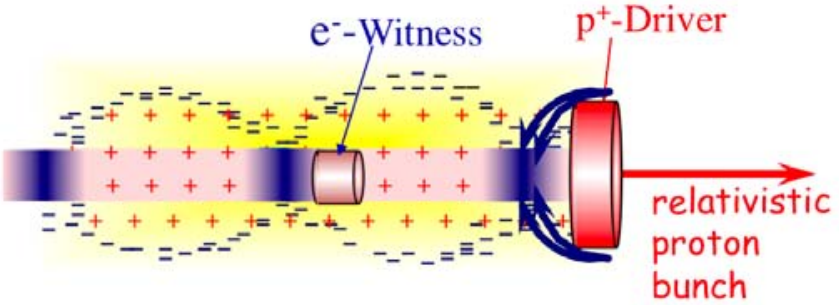

(c)

Fig. 1. (a) The PWFA principle: a drive beam drives a wake in a plasma. Part of the energy lost to the plasma can be used to accelerate a trailing beam. (b) When a drive beam is depleted and cannot drive the plasma anymore, it is ejected from the beam line and a new drive bunch can be injected into a new plasma stage. PD-PWFA has the potential for providing very-high-energy gain in a single stage. (c) A short proton bunch can generate a plasma wake structure similar to the electron-driven blowout regime, a structure ideal for accelerating electrons.

Following the successful demonstration of high-gradient acceleration in electron-beam-driven PWFA [4], ideas to use energetic proton beams as drivers came about - first written down in Refs. 12 and 13, illustrated in Fig. 2 and discussed in more detail below. Due to the heavy mass of protons and consequently low synchrotron radiation losses, it is possible to accelerate protons to very high energies in synchrotrons. In a single proton-driven PWFA stage, electron or positron bunches can potentially be accelerated to energies of the same order as the proton energy, without the need for high transformer ratios. A positively charged drive bunch pulls plasma electrons toward the axis as it propagates [14], as opposed to an electron drive bunch, which blows the plasma electrons away from the axis. If the electron drive current is large enough, plasma electrons may be completely blown away during the passage of the driver, forming an accelerating cavity as the blown-out plasma electrons are attracted back toward the ions; see Fig. 1(a). This operating regime is commonly known as the blowout regime and has been well studied for electron drivers, both theoretically and experimentally [4], and is ideal for

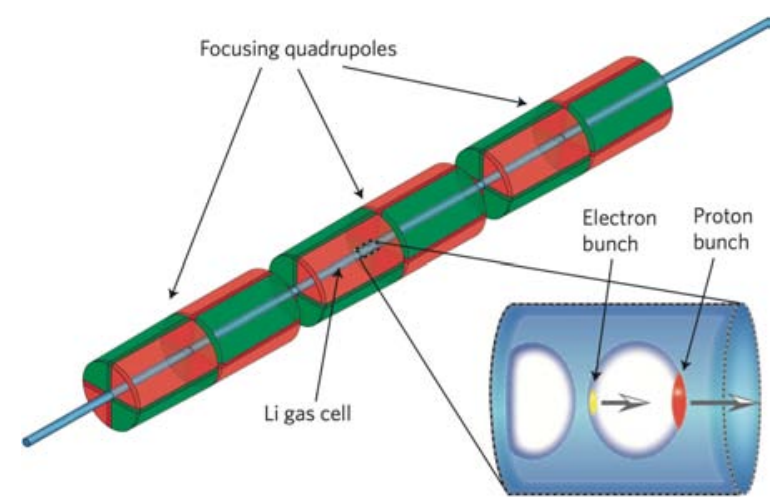

Fig. 2. Illustration of a PD-PWFA concept; a compressed version of the LHC bunch accelerates an electron bunch to $620 \mathrm{GeV}$ in a single, $450-\mathrm{m}$-long plasma stage, with external focusing quadrupoles. (From Ref. 13.) 
accelerating electrons. If the positively charged driver is sufficiently short, the plasma electrons will mostly overshoot behind the drive bunch and form a plasma cavity structure similar to that of an electron driver, as discussed in Refs. 13 and 15 and illustrated in Fig. 1(c). This is true for both positron and proton bunches. High-energy proton bunches are more easily and more efficiently produced than positron ones. An ideal drive beam for accelerating electron bunches to high energy in a single stage is therefore a short proton bunch with both high particle energy and sufficient total bunch energy.

\section{High-Energy Proton Drivers}

High-energy proton bunches produced by synchrotrons are available at CERN in Switzerland [16], Fermilab in the USA [17], J-PARC in Japan [18] and IHEP in Russia [19]. Table 1 summarizes the main beam parameters of proton synchrotrons potentially of interest for proton-driven PWFA (PD-PWFA), here defined as beams with a particle energy of at least $10 \mathrm{GeV}$. Figure 3 shows the CERN accelerator complex, which produces three of the highest-energy proton beams currently available; the Proton Synchrotron (PS), Super Proton Synchrotron (SPS) and Large Hadron Collider (LHC) beams. The LHC provides the highest particle energy available worldwide $(7 \mathrm{TeV})$, while J-PARC provides the highest total bunch energy $(130 \mathrm{~kJ})$.

\subsection{Generating efficient drivers}

The scale of accelerating fields in plasmas is given by the wave-breaking field, of strength $\sim \sqrt{n_{0} / 10^{16} \mathrm{~cm}^{-3}} 10 \mathrm{GV} / \mathrm{m}[20]$. In order to obtain accelerating fields of order $\mathrm{GV} / \mathrm{m}$ in PWFA, the plasma density needs to be $\gtrsim 10^{14} \mathrm{~cm}^{-3}$, with the corresponding plasma wavelength $\lambda_{\text {pe }} \lesssim$ few mm. This requires drive beam lengths, $k_{\mathrm{pe}} \sigma_{z} \sim \sqrt{2}$, to be of the order of a few hundred $\mu \mathrm{m}$ (duration of ps). Since available high-energy proton beams have bunch lengths of the order of a few hundred mm (duration of ns), these bunches are several orders of magnitude too long to efficiently drive a plasma wake. Available proton bunches are long because until now there has been little need for short bunches, and because instabilities in synchrotrons increasingly become a problem with increasing beam current. To generate drivers for PD-PWFA, it has been suggested to either compress the entire beam or microbunch the beam in order to resonantly drive the wake. Both options are discussed in the following.

\subsubsection{Bunch compression}

In Ref. 16 a number of options for bunch length manipulation in synchrotrons are studied, including bunch rotation prior to extraction, for example by lattice manipulation and $\mathrm{RF}$ voltage changes, and $4 \mathrm{D}$ or $6 \mathrm{D}$ emittance exchange transformations, possibly involving fast-deflecting crab cavities. Reference 16 concludes that a single method is probably not sufficient to reduce the bunch length from the existing ns lengths to ps length, and would probably need to be combined with bunch compression after extraction from the synchrotron. After extraction, the bunch can be shortened in a magnetic bunch compressor, as is common for electron bunches. First, RF voltage

Table 1. Proton beams of $10 \mathrm{GeV}$ or higher energy worldwide.

\begin{tabular}{|c|c|c|c|c|c|}
\hline Synchrotron & $\begin{array}{c}\text { Maximum } \\
\text { particle energy } \\
(\mathrm{GeV})\end{array}$ & $\begin{array}{c}\text { Maximum } \\
\text { bunch energy } \\
(\mathrm{kJ})\end{array}$ & $\begin{array}{l}\text { Minimum } \\
\text { bunch duration } \\
\text { (ns) rms }\end{array}$ & $\begin{array}{l}\text { Relative } \\
\text { energy spread } \\
\text { (\%) rms }\end{array}$ & $\begin{array}{l}\text { Transverse } \\
\text { emittance } \\
\text { ( } \mathrm{mm} \text { mrad) }\end{array}$ \\
\hline $\begin{array}{l}\text { CERN PS, } \\
\text { Geneva, Switzerland }\end{array}$ & 26 & 0.54 & 1 & 0.1 & 3.0 \\
\hline $\begin{array}{l}\text { CERN SPS, } \\
\text { Geneva, Switzerland }\end{array}$ & 450 & 8.3 & 0.4 & 0.03 & 3.5 \\
\hline $\begin{array}{l}\text { CERN LHC, } \\
\text { Geneva, Switzerland }\end{array}$ & 7,000 & 124 & 0.25 & 0.01 & 3.75 \\
\hline $\begin{array}{r}\text { Fermilab Main Injector, } \\
\text { Batavia, Illinois, USA }\end{array}$ & $120(150)$ & 2.1 & 0.6 & 0.04 & 5.4 \\
\hline $\begin{array}{l}\text { J-PARC, } \\
\text { Ibaraki prefecture, Japan }\end{array}$ & 30 & 130 & 15 & 0.05 & 1 \\
\hline $\begin{array}{l}\text { IHEP U70, } \\
\text { Protvino, Russia }\end{array}$ & $50(70)$ & $4.0(5.6)$ & 12 & 0.1 & 2 \\
\hline
\end{tabular}




\section{CERN's Accelerator Complex}

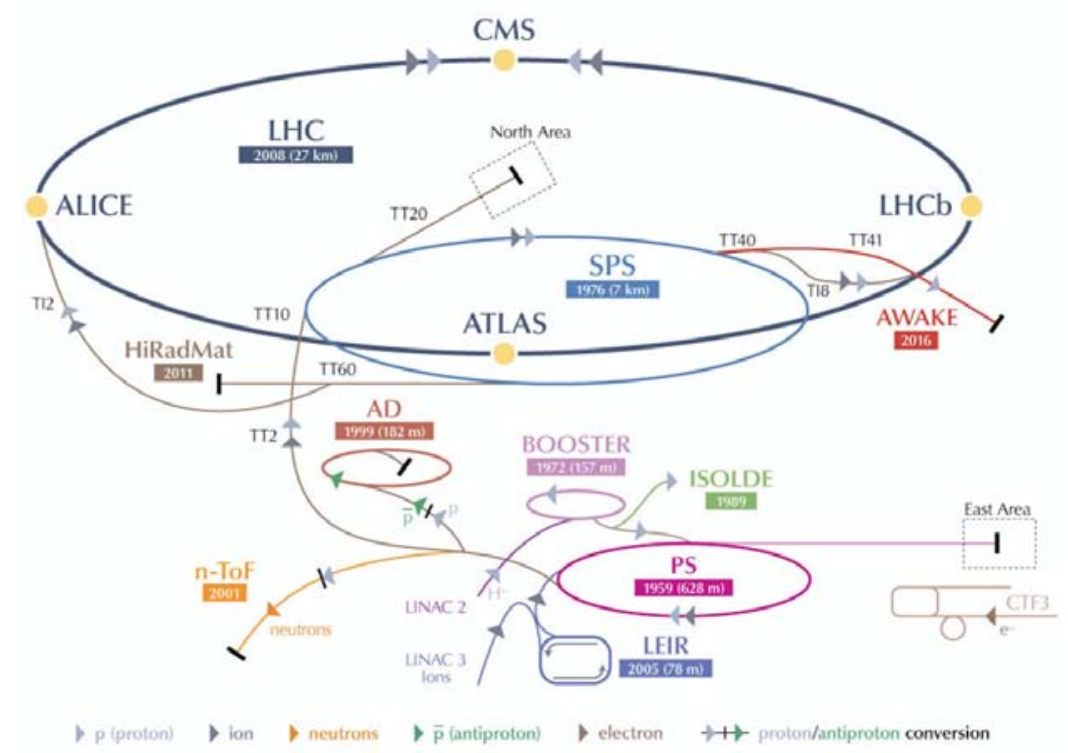

Fig. 3. The CERN accelerator complex produces three of the highest-energy proton beams currently available: the PS, SPS and LHC beams. The LHC bunches provide the highest particle energy available worldwide ( $7 \mathrm{TeV}$ design energy). (Figure courtesy of CERN.)

is used to introduce an energy modulation varying along the bunch. Then, particles follow dispersive paths in a magnetic chicane or dogleg, compressing the bunch longitudinally, while increasing the uncorrelated energy spread. In Ref. 21 it is estimated that to compress a $1 \mathrm{TeV}$ beam from $1 \mathrm{~cm}$ to $165 \mu \mathrm{m}$ would require $65 \mathrm{GV}$ of $\mathrm{RF}$ voltage, and in Ref. $22,25 \mathrm{GV}$ of $\mathrm{RF}$ voltage is estimated to compress the CERN SPS bunch from $1 \mathrm{~cm}$ to $350 \mu \mathrm{m}$. Figure 4 illustrates different compressions methods. While bunch compression of high-energy proton bunches may be interesting for future PD-PWFA applications, this would not necessarily be the preferred choice for the first PD-PWFA experiments, due to the size and cost of the RF system and magnetic compressors.

\subsubsection{Microbunching and self-modulation}

An effective drive beam can also be produced by microbunching of an initially long bunch, so that the wake of each microbunch adds to those set up by leading bunches $[3,10,23]$. The microbunching

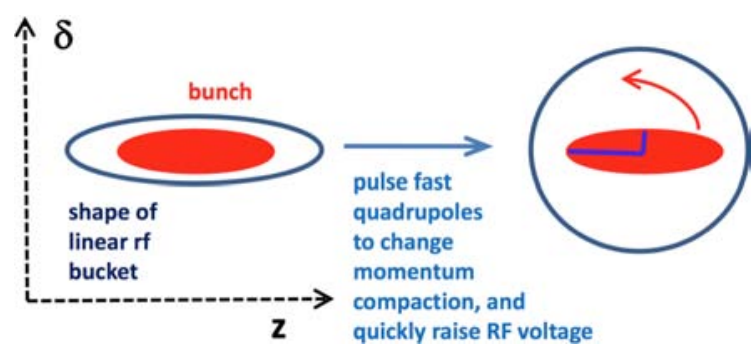

(a)

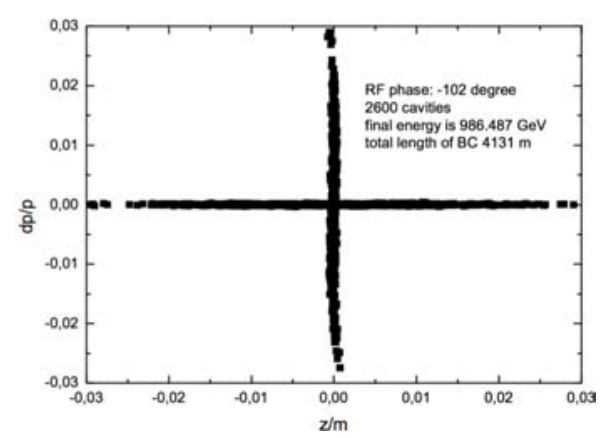

(b)

Fig. 4. Proton bunches can be compressed by a number of different methods, such as (a) fast bunch rotation prior to extraction from the synchrotron, or (b) magnetic bunch compression after extraction; the plot shows the longitudinal phase space before (flat) and after (upright) compression. Even when one is combining different methods, the RF voltage required to generate proton drivers of ps duration would imply large and costly compression systems. [Figure (a) from Ref. 16; Fig. (b) from Ref. 21.] 
spacing must be $\sim \lambda_{\text {pe }}$, in order to drive the wake resonantly. We first discuss the concept of selfmodulation, as this has a particular interest for PDPWFA. It was understood early that a transverse two-stream instability [24] could radially microbunch a long beam propagating in a plasma [25], with bunching period $\sim \lambda_{\text {pe }}$ - a process called the selfmodulation instability (SMI). The equations for the SMI of a particle beam in a plasma are analogous to those of a laser beam in plasma, extensively studied in for example Refs. 26 and 27. The promise of PDPWFA [13], combined with the technical challenges of compressing existing proton bunches to lengths of $\sim k_{\mathrm{pe}}^{-1}$, leads to a renewed interest for the SMI in a particle beam. In Refs. 28 and 29 an analytical model for the SMI is developed, and mathematical expressions for the instability growth rates are provided. It is shown by numerical simulations that the microbunches generated by the SMI have the correct bunch-to-bunch distance to resonantly excite a large amplitude wake $(\sim \mathrm{GV} / \mathrm{m})$. It is furthermore shown that the SMI can be seeded, either by a precursor bunch [25] or by using a charge profile with a hard leading edge [28]. In order to synchronize the wakefields with an injected witness bunch, seeding is important. Also, if the SMI is not seeded the competing transverse hosing instability [24] may destroy the coherence of the plasma wakefields, strongly limiting the amplitude of the fields. References 28 and 30 show that by seeding the SMI the growth rate of the hosing instability may be greatly suppressed, with the wakefields mostly unperturbed. Figure 5, from Ref. 28, illustrates the effect of seeding by using a hard-edge half Gaussian as charge profile. For a regular Gaussian charge profile, the on-axis longitudinal field is suppressed by a factor of 5 with respect to the half Gaussian, as the former is subject to the competing hosing instability. Due to the simplicity of the SMI bunching scheme, where no extra bunching hardware or compression hardware is required, this concept was adopted for the first proposals for PD-PWFA experiments [31, 32].

In numerical simulations with self-modulated drivers, it was observed that although a large amplitude wakefield is indeed produced once the SMI has developed, the proton beam continues to evolve as it propagates, eventually leading to decay of the longitudinal field [33]. It was furthermore observed that by having a step in the plasma density of a few

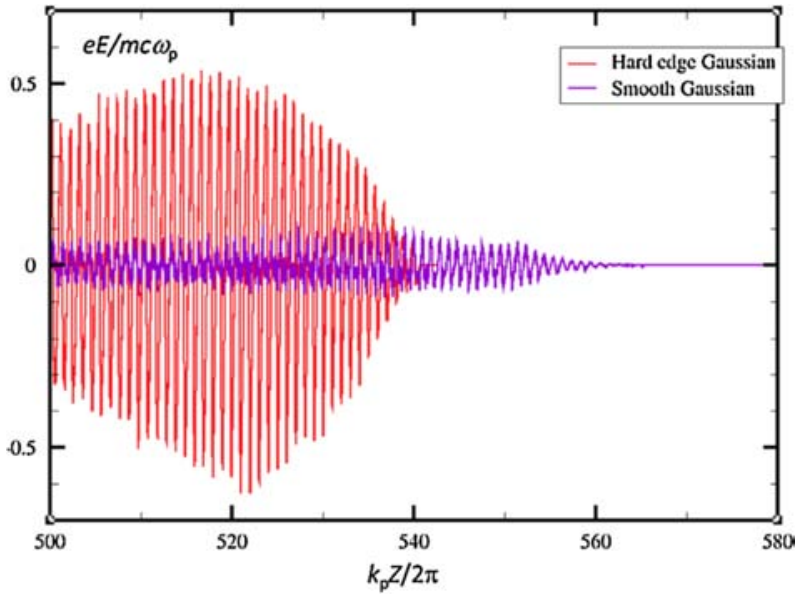

Fig. 5. The axial longitudinal wakefield in a plasma (the beam travels toward the right): in purple, for a regular Gaussian charge profile: in red, for a hard-edge half Gaussian where the cut seeds the self-modulation instability. Because of the competing hosing instability, the field generated by the regular Gaussian is suppressed by a factor of 5 with respect to hard-edge half Gaussian. (From Ref. 28.)

percent, in the region where the SMI is developing, the proton beam evolution can be partly stabilized and the field decay mitigated [33]. Later work [34, 35] discuss the optimal location and amplitude of such a step as a function of train length, beam emittance and other parameters. However, whether a stepped plasma density profile is optimal to best stabilize the proton beam evolution is not fully understood. The field amplitudes arising from self-modulated beams, as well as the efficiency of the step, are parameterdependent. In Ref. 36 the accelerating fields generated by the CERN PS, SPS and LHC beams are compared, both without and with a density step, and the results are summarized in Fig. 6. With a stepped plasma density profile, the wake from the LHC beam stabilizes at a field amplitude of about $1 \mathrm{GV} / \mathrm{m}$, and can efficiently drive a wake for many kilometers, according to Ref. 36. These simulations were performed using the hybrid code LCODE [37, 38]. It was found that the peak fields are roughly proportional to the number of particles in the drive bunch and inversely proportional to the square of the transverse bunch size, which is in good correspondence with theory $[5,34]$. However, in some cases, as discussed in Ref. 39, the wakefield amplitude is limited by nonlinear elongation of the wave period.

Alternatively, a train of microbunches with the desired frequency can be generated by longitudinal 


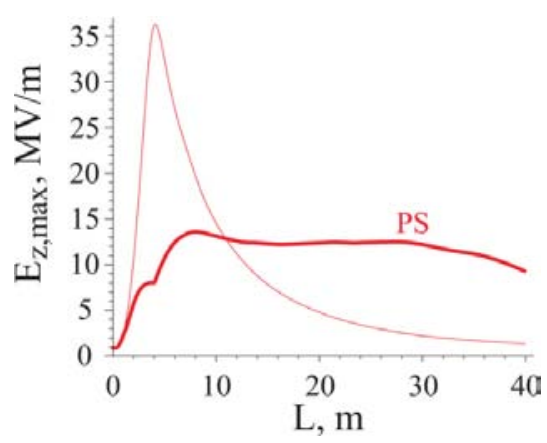

(a)

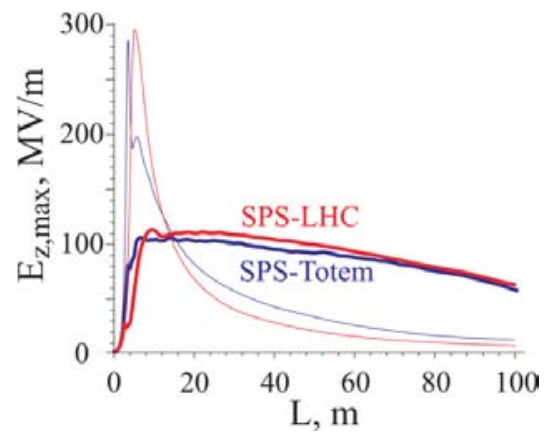

(b)

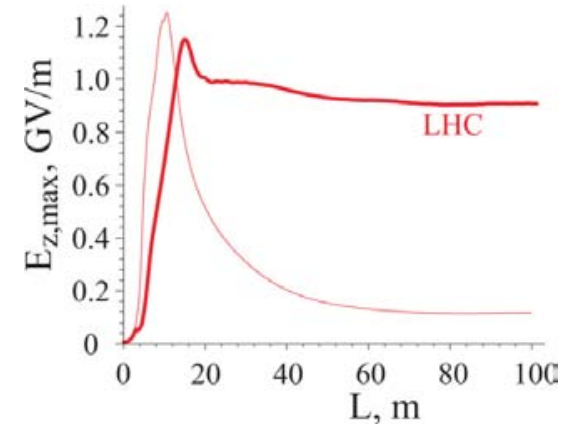

(c)

Fig. 6. The peak axial accelerating field from self-modulated proton bunches: (a) CERN PS, (b) CERN SPS and (c) CERN LHC. The thin lines show the fields for a uniform plasma profile, while the bold lines show the field when the plasma profile has a density increase in a step of a few percent after 1-3 $\mathrm{m}$ of propagation. This density step aids in stabilizing the proton beam evolution and the accelerating fields. All simulations are seeded by using a hard-cut half Gaussian charge profile. (From Ref. 36.)

bunching of the proton beam before it enters the plasma. A number of methods have been studied for generating microbunched drive trains, also because this is an important topic for free electron lasers. They include energy modulation followed by velocity bunching or magnetic compression, driving a photocathode with a pulsed laser train ("comb-laser") [41], or using metal masks to carve out a bunch pattern in one of the transverse dimensions, typically at low energy. After the mask, the transverse modulation is converted into a longitudinal modulation by either introducing correlated energy spread and high dispersion where the mask is located [42], or using emittance exchange lattices [43]. Longitudinal microbunching has the following advantages over self-modulation: it can provide more control over the initial bunch phase, there is no need to rely on an instability to grow in the plasma, and a larger percentage of the initial charge can be used to drive the wake. On the other hand, longitudinal bunching would require hardware upgrades of the existing facilities, for example large amounts of new RF power. Most of the above methods are not mature enough to be readily used without further R\&D. For PD-PWFA applications, the plasma wavelength must be enforced to match the longitudinal bunching wavelength, putting constraints on the plasma density tolerances. Microbunching by selfmodulation has the advantage that the bunch spacing is automatically adjusted to drive the plasma resonantly. The evolution over long distances of both self-modulated and longitudinally bunched trains is being investigated. Which option will work better in practice to generate the most efficient PD-PWFA drivers remains to be studied experimentally.

\section{PD-PWFA Experiments}

The first written concept of a PD-PWFA accelerator, "Design for an Advanced Accelerator Driven by a Proton (or Ion) Beam," is from 2003 [12]. In this work it is proposed to use a $1 \mathrm{TeV}$ proton beam to accelerate electron or positron beams up to an energy of $500 \mathrm{GeV}$ or more. 2D numerical simulations were performed assuming a short proton beam. The challenge of compressing available Fermilab proton beams to the required lengths was acknowledged. This work was not pursued further. A few years later, in 2009, Ref. 13 discussed various aspects of PD-PWFA, also assuming a short bunch of $100 \mu \mathrm{m}$ length. Acceleration of $600 \mathrm{GeV}$ electrons using a $1 \mathrm{TeV}$ proton bunch was demonstrated by $2 \mathrm{D}$ and 3D numerical simulations. Strong external focusing quadrupoles were used to mitigate emittance expansion of the drive beam head. In Ref. 36 numerical studies showed that the nominal-length LHC beam, after undergoing self-modulation, could also accelerate a test electrons up to $6 \mathrm{TeV}$, without the use of external focusing quadrupoles. Following the promise of PD-PWFA in Refs. 13 and 36, a protocollaboration studied various options for drive beam and site locations, and a Letter of Intent for a "Demonstration Experiment in Proton-Driven Plasma Wakefield Acceleration" [31] was submitted to the CERN SPS 
and PS experiments committee (SPSC) in May 2011, with the aim of demonstrating PD-PWFA using the CERN SPS beam, relying on the self-modulation instability to produce an efficient driver. The experiment, named "The Advanced Proton-Driven Plasma Wakefield Acceleration Experiment," or AWAKE, was approved by the CERN SPSC and is at the time of writing undergoing commissioning. AWAKE is the only approved PD-PWFA experiment worldwide and will be discussed in more detail below.

PROTOPLASMA [32] at Fermilab was a proposed $R \& D$ project with many similarities to the AWAKE proposal [31], suggesting to use the $120 \mathrm{GeV}$ Fermilab Main Injector beam as a self-modulated proton driver. This proposal was not approved and there are currently no proposals to perform PDPWFA experiments at Fermilab. In Asia and Russia there are also currently no plans or proposals to perform PD-PWFA experiments.

\subsection{Proof-of-principle studies}

Proposed and ongoing PD-PWFA experiments depend on the self-modulation instability to generate a microbunch drive train from a long proton bunch. Also, electron or positron beams, long with respect to the plasma wavelength, may self-modulate in a plasma $[44,45]$. Several experiments have been proposed and performed in order to study the SMI in electron and positron beams, since such beams have been more readily accessible at accelerator test facilities.

At the Brookhaven National Laboratory Accelerator Test Facility (ATF), an experiment to study the SMI as well as the seeding of the instability was performed [44]. In the experiment a $58 \mathrm{MeV}$ electron bunch, about $1 \mathrm{~mm}$ long with a sharp rising edge, was sent into plasmas of varying densities, corresponding to 1-7 plasma wavelengths along the bunch. Evidence of energy modulation at the plasma wavelengths was demonstrated [46], as shown in Fig. 7(a). It was also shown that the sharp rising edge of the bunch seeds the phase of the wakefield, a necessary condition for the deterministic external injection of a witness bunch in an accelerator. The results show that the seeding of the instability is possible since the transverse wakefields necessary for the SMI seeding are always associated with the longitudinal ones observed in the experiment.

In Ref. 45 the SMI in the $20 \mathrm{GeV}$ electron and positron beams available at the FACET test facility [47] is studied numerically, showing that the beams may self-modulate if $\sigma_{z} \gg \lambda_{\text {pe }}$, and if the SMI is seeded in order to suppress the competing hosing instability. Experiments with the target of demonstrating the SMI in electron and positron

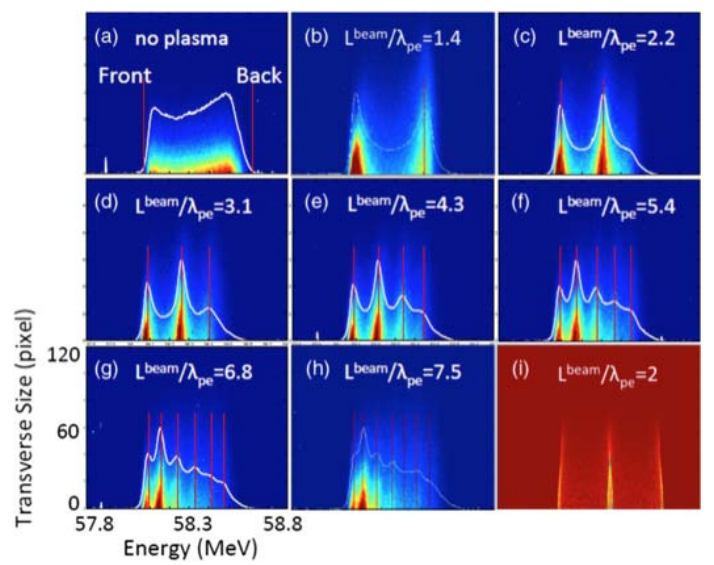

(a)
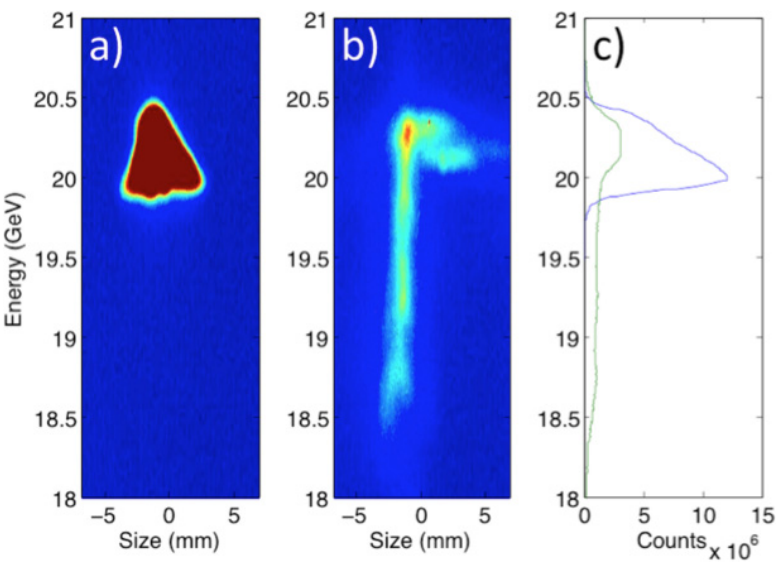

(b)

Fig. 7. Proof-of-principle studies which aim at demonstrating the self-modulation instability in lepton beams, performed with long electron and positron bunches where $k_{\mathrm{pe}} \sigma_{z} \gg 1$. (a) At ATF/BNL, evidence of energy modulation along a long bunch, at the plasma wavelength, was observed. The experiment also demonstrated that a steeply rising charge profile seeded the fields, ensuring a deterministic phase reference [46]. (b) At FACET/SLAC, energy loss one order of magnitude stronger than that expected from linear theory was observed - about $2 \mathrm{GeV}$ and about $100 \mathrm{MeV}$, respectively [48]. The measured $2 \mathrm{GeV}$ deceleration is in reasonable agreement with numerical simulations. (c) The plasma source at PITZ/DESY, where experiments are underway with the aim of producing direct evidence of the SMI of electron beams [49]. (Figures from Refs. 46, 48 and 49.) 


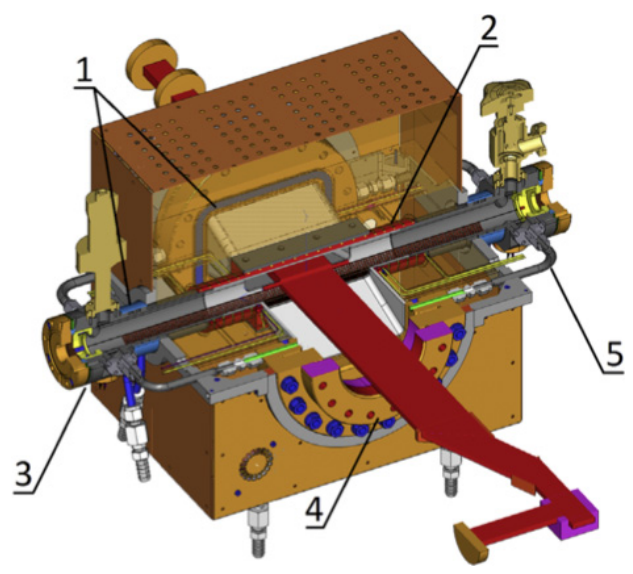

(c)

Fig. 7. (Continued)

beams at FACET were performed [48]. An about 1.5-mm-long bunch was used. The results [48] show that the energy spectra and the halo formation of a long electron bunch, having interacted with lithium plasma, are consistent with a bunch that has selfmodulated. In particular, energy loss one order of magnitude stronger than that expected from linear theory was observed, about $2 \mathrm{GeV}$ versus about $100 \mathrm{MeV}$, as illustrated in Fig. 7(b). The $2 \mathrm{GeV}$ deceleration is in reasonable agreement with the predictions of the simulated self-modulation in Ref. 45. However, no direct observation of the SMI was reported in Ref. 48.

At the Photo Injector Test facility at DESY Zeuthen (PITZ), complementary experiments are underway to demonstrate self-modulation of an electron beam [49]. In 2015 a first set of experiments was conducted utilizing a novel cross-shaped lithium heat pipe oven and an ArF excimer laser for plasma generation. No self-modulation was observed in the first experimental run [49], for reasons thought to be related to plasma density and low ionization laser energy. The experiment is planned to continue in 2016, with an upgraded plasma cell and ionization laser beam to address shortcomings of the first experiment. Figure 7(c) shows a schematic of the PITZ plasma source, from Ref. 49.

Additional simulation studies related to PDPWFA have been performed by various groups. Due to the long drive beams proposed for PDPWFA, where wakefields are used after many plasma oscillations, ion motion is potentially a bigger concern for PD-PWFA than for electron-driven PWFA.

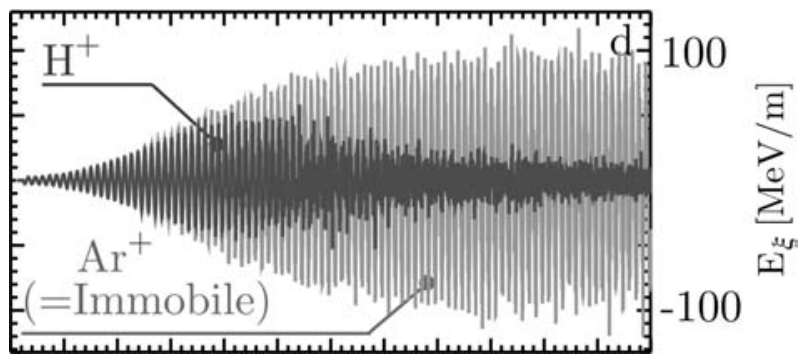

Fig. 8. The effect of ion motion in PD-PWFA, illustrated for the AWAKE parameter. For a hydrogen plasma the ion motion suppresses the resonant wake buildup (the beam travels toward the left). For an argon plasma the effect of the ions on the field is negligible. (From Ref. 50.)

References 50 and 51 show that the growth rate of the SMI, for AWAKE parameters, is reduced when using a hydrogen plasma, and unaffected when using an argon plasma. (See Fig. 8). Reference 52 indicates that the SMI may develop even if the plasma channel is narrow, $k_{\mathrm{pe}} \sigma_{r}>1$, and also in transversally inhomogeneous plasma channels. References 54 and 55 investigate proton-driven hollow channel acceleration. Recently, PD-PWFA study activities have also been initiated in South Korea [56]. In addition extensive simulation studies have been performed as preparation for the AWAKE experiment. These will be discussed in the next part of this review.

\section{AWAKE}

We now turn our attention to AWAKE, an experiment at CERN aimed at studying the driving of plasma wakefields with high-energy proton bunches [57-59]. 


\subsection{Overview}

As mentioned above, proton bunches are interesting plasma wakefield drivers because existing bunches carry a large amount of energy per particle (many $\mathrm{GeV}$ ) and per bunch (many kilojoules). Staging of many plasma cells may therefore not be required for the witness bunch (electrons or positrons) to reach the TeV-energy scale [13]. However, high-energy proton bunches also have a large rigidity, which means that it is difficult to make them either very short or very tightly focused. This limits the plasma density that can be used in a PWFA since the bunch and plasma density must satisfy $k_{\mathrm{pe}} \sigma_{z} \simeq \sqrt{2}$ and $k_{\mathrm{pe}} \sigma_{r} \lesssim 1$ for effective wakefield driving. AWAKE therefore aims at reaching accelerating fields on the order of $1 \mathrm{GV} / \mathrm{m}$ in long plasmas $(>10 \mathrm{~m})$ with the electron density in the $10^{14} \mathrm{~cm}^{-3}$ to $10^{15} \mathrm{~cm}^{-3}$ range. Since available proton bunches are long, the $k_{\mathrm{pe}} \sigma_{z} \simeq \sqrt{2}$ condition is fulfilled by using the SMI, as explained above.

The first experiments will therefore focus on the study of the SMI of the proton bunch in the plasma (2016-17). Later experiments will use externally injected electrons to sample the wakefields and to be accelerated to the GeV-energy scale.

The AWAKE schematic is shown in Fig. 9, and numbers in the following text refer to numbers on the figure.

The AWAKE experiment uses the long proton bunch $\left(\sigma_{z} \simeq 12 \mathrm{~cm}\right)$ produced by the CERN SPS, with a proton energy of $400 \mathrm{GeV}$. The proton bunch extracted from the SPS (1') is merged with the ionizing laser pulse. The laser pulse and the proton bunch travel together through the metal vapor cell (3-4). The proton bunch transverse size at the plasma entrance is $\sigma_{r} \simeq 220 \mu \mathrm{m}$. The laser pulse transverse size is around $1 \mathrm{~mm}$. The copropagating ionization front shown in (2) provides the seeding of the SMI. Only the fraction of the bunch participating in SMI (behind the laser pulse, in the plasma) and wakefield excitation is shown in (1') and (4'). Growth of the SMI and the resulting self-modulation of the proton bunch occurs over the first 3-5 $\mathrm{m}$ of plasma (3). The modulated bunch (4') resonantly drives wakefields over the remaining length of plasma (4). The laser pulse is dumped (5) and the proton bunch radial modulation is measured using electro-optic sampling (EOS) diagnostics (6) and optical transition radiation diagnostics (7). An RF gun driven by a laser pulse derived from the ionizing laser system produces a witness electron bunch (8). The electron bunch can be injected into the wakefields, at an adjustable position in the plasma (9). After the plasma, the electron bunch energy spectrum is measured using a broad acceptance magnetic spectrometer (10).

Simulations show that, with the long proton bunch and the SMI, wakefields with amplitudes close to $1 \mathrm{GV} / \mathrm{m}$ can be excited in a plasma with a typical density of $7 \times 10^{14} \mathrm{~cm}^{-3}[36,59,60]$. The same simulations show that the electrons reach an energy of around $1 \mathrm{GeV}$ (from their $16 \mathrm{MeV}$ injection energy) with a finite $(<10 \%)$ energy spread.

\subsubsection{Beam and site choice}

The experimental as well as the physics parameters are dependent on the beams and infrastructure

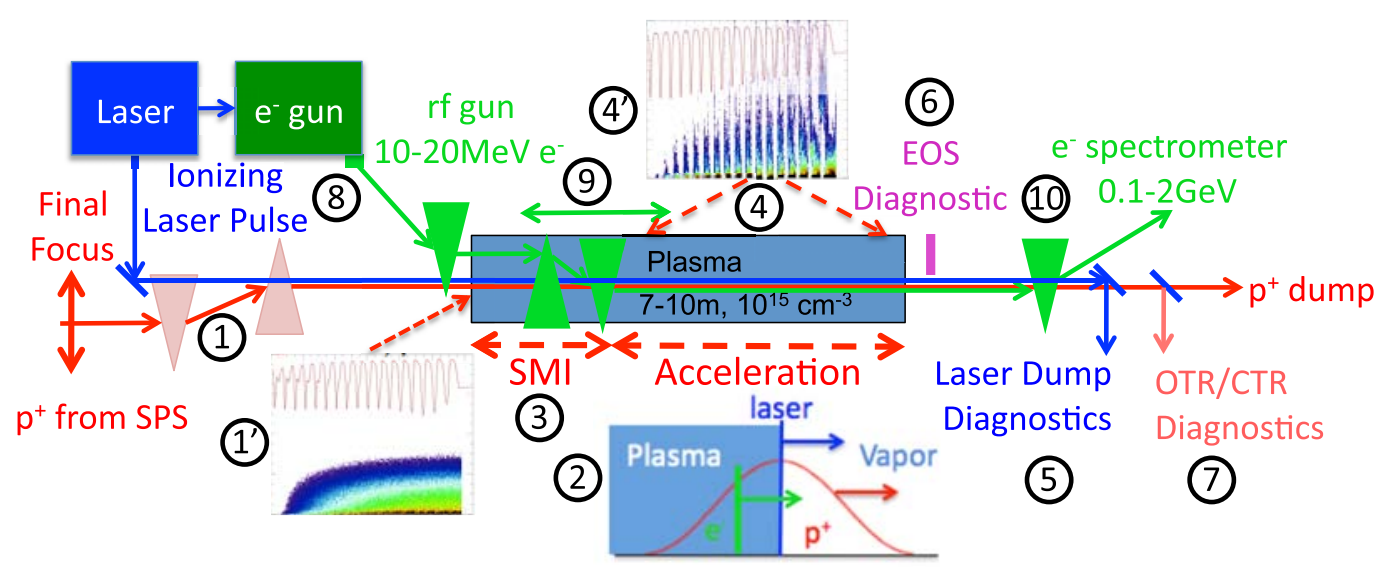

Fig. 9. Schematic of the AWAKE experiment. Numbers are used in the text. 
available at CERN. Numerical simulations [36, 61] indicate that higher-energy bunches generally produce larger accelerating gradients over longer distances. Beams produced by the CERN PS at $24 \mathrm{GeV}$ were therefore excluded. The beams produced by the CERN SPS at $400 \mathrm{GeV}$ were chosen because of the lack of access to the LHC beams.

A number of sites around the SPS were considered and the facility previously known as CERN Neutrinos to Gran Sasso (CNGS) was chosen. It required a minimum of beam line and facility modifications to become the AWAKE facility and was also the financially most advantageous option still fulfilling the AWAKE requirements [58]. A schematic of the location of AWAKE is shown in Fig. 10.

\subsubsection{Proton bunch}

The AWAKE proton energy is $400 \mathrm{GeV}$, limited by the bending magnets in the transfer line to the experimental area. A single bunch has a population of $1-3 \times 10^{11}$ particles and an rms length of $\sigma_{z}=12 \mathrm{~cm}$. The nominal beam normalized emittance is $3.6 \mathrm{~mm}$-mrad and the relative energy spread $0.03 \%$. With available quadrupole magnets the beam can be focused to $\sigma_{r}=220 \mu \mathrm{m}$, equivalent to a beam beta function at its waist, $\beta^{*}$, of $4.5 \mathrm{~m}$. The proton will initially be delivered to AWAKE every $30 \mathrm{~s}$, with the possibility of being delivered every $7.2 \mathrm{~s}$.

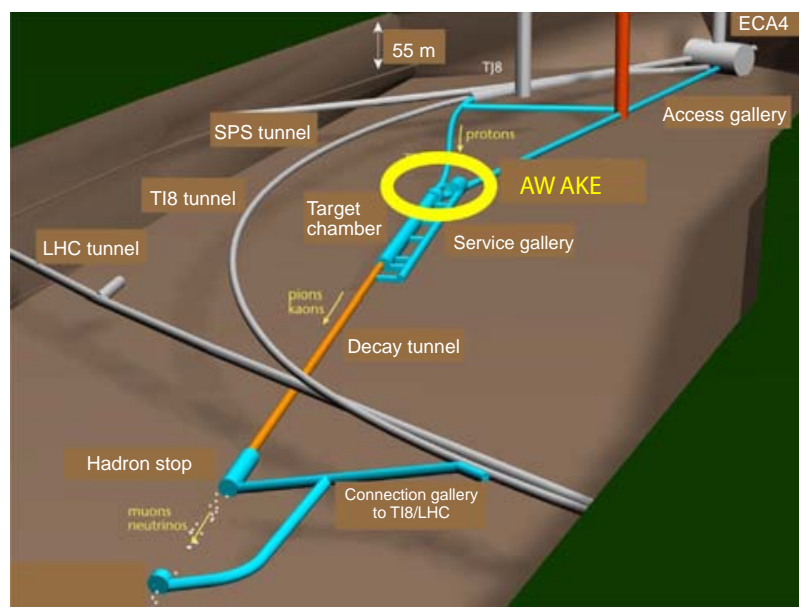

Fig. 10. Schematic of the AWAKE location at CERN. A single proton bunch is extracted from the SPS near one of the LHC extraction lines (TJ8). The plasma is located just upstream of the ex-CNGS target chamber location. The service gallery is used for AWAKE electronics and diagnostics because of the lower radiation level.

\subsubsection{Plasma}

The plasma parameters follow from the bunch parameters. To satisfy $k_{\mathrm{pe}} \sigma_{r}<1$, the plasma electron density must not exceed $7 \times 10^{14} \mathrm{~cm}^{-3}$. At the nominal electron plasma density of $7 \times 10^{14} \mathrm{~cm}^{-3}$, the relativistic plasma wave period is $\lambda_{\text {pe }} \simeq 1.3 \mathrm{~mm}$ and the plasma frequency $f_{\mathrm{pe}}=237 \mathrm{GHz}$. Since $\lambda_{\mathrm{pe}} \ll \sigma_{z}$, the relativistic proton bunch is subject to the SMI, which will modulate its density with $\mathrm{a} \simeq \lambda_{\text {pe }}$ longitudinal period. The initial beam-to-plasma-density ratio is $n_{b 0} / n_{e} \simeq 6 \times 10^{-3}$. The typical peak accelerating field that can be expected (with a single short bunch) is on the order of $\frac{n_{b 0}}{n_{e}}$ times the wavebreaking field, $\sim \sqrt{n_{0} / 10^{16} \mathrm{~cm}^{-3}} 10 \mathrm{GV} / \mathrm{m}$. This corresponds to $\simeq 14.5 \mathrm{MV} / \mathrm{m}$. However, since $N_{\lambda} \simeq$ $\sigma_{z} / \lambda_{\text {pe }}$ bunches drive the wakefields, accelerating fields on the order of $1.4 \mathrm{GV} / \mathrm{m}$ can be expected.

The plasma length is chosen to be $10 \mathrm{~m}$ or about $2 \beta^{*}$. The plasma radius must be larger than the extent of the wakefields, although small-radius plasma may not strongly affect the SMI development [52]. The minimum plasma radius is $1 \mathrm{~mm}$, on the order of $\lambda_{\text {pe }}$.

The SMI bunch train resonantly drives the plasma wakefields. This imposes stringent conditions on the plasma density uniformity and profile. While the SMI is relatively insensitive to plasma density variations $[62,63]$, the acceleration of witness particles over a long plasma is not. To be effectively accelerated the witness particles must remain within the accelerating and focusing phase of the wakefields, i.e. within about one quarter of a plasma period. A variation in plasma density $\delta n_{e}$ translates into a variation of the plasma wavelength of $\delta \lambda_{\mathrm{pe}}=-\frac{1}{2} \lambda_{\mathrm{pe}} \frac{\delta n_{e}}{n_{e}}$. The variation in the relative position of the witness particles $N_{\lambda} \delta \lambda_{\text {pe }}$ must remain small compared to the extent of the accelerating and focusing region of the wakefields, i.e. $N_{\lambda} \delta \lambda_{\text {pe }} \ll \lambda_{\text {pe }} / 4$. With the numbers above, this means that the plasma density uniformity must remain within $0.2 \%$ [62].

On-axis external injection of witness particles also imposes a condition on the plasma density ramp at the plasma entrance that will exist in actual plasma sources. Since the plasma electron density varies in the ramp, so does the period of the wakefields. The injected particles therefore find themselves in defocusing regions of the wakefields and exit the wakefields. Simulation results show that, with AWAKE parameters, when the injected 
particles cover the entire length of a wakefield period, most injected electrons are lost if the plasma density ramp length exceeds about $10 \mathrm{~cm}[45,60]$. The case of injected $e^{+}$is slightly different, but the capture efficiency remains small $(\sim 5 \%)$. Note that a sharp density ramp is especially important at the plasma entrance, where the injected electrons have a low energy $(10-20 \mathrm{MeV})$.

\subsubsection{SMI development and seeding}

As mentioned before, the transverse hosing instability is of concern because it has a growth rate similar to that of the SMI $[28,30,45]$. Due to the simulation challenges related to the AWAKE parameters, in particular the very long length of the drive bunch in terms of plasma skin depth $c / \omega_{\text {pe }}$, no systematic study of the relative importance of the hosing instability with respect to SMI has been performed. However, simulation results indicate that without seeding the SMI does not develop to significant wakefield values over the AWAKE plasma length. SMI seeding is therefore mandatory for AWAKE and is included in the plasma source that is described next.

\subsection{Plasma source}

The plasma source that was chosen for AWAKE is a laser-ionized, rubidium $(\mathrm{Rb})$ vapor source [65]. Figure 11 shows a schematic of the $\mathrm{Rb}$ source. $\mathrm{Rb}$ has a relatively low ionization potential $(4.177 \mathrm{eV})$ for its first electrons and can therefore be fieldionized at laser pulse intensities larger than only $1.7 \times 10^{12} \mathrm{~W} / \mathrm{cm}^{2}$. Field ionization frees the first electron of every Rb atom, thereby making the plasma electron density (and thus its uniformity) equal to that of the neutral vapor.

\subsubsection{Density uniformity}

$\mathrm{Rb}$ melts at $39.30^{\circ} \mathrm{C}$. A vapor density $n_{\mathrm{Rb}}$ in the $1-10 \times 10^{14} \mathrm{~cm}^{-3}$ range is obtained by heating $\mathrm{Rb}$ to $150-200^{\circ} \mathrm{C}$, corresponding to a vapor pressure of $0.002-0.02$ mbar. The vapor density uniformity is obtained by imposing a very uniform temperature along the vapor column (without flow). This is obtained by using a heat exchanger with a large heat transfer fluid flow. Figure 12 shows a picture of the heat exchanger as installed in the AWAKE facility. Measurements have shown that the temperature

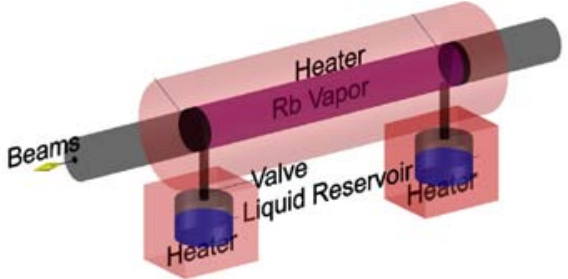

(a)

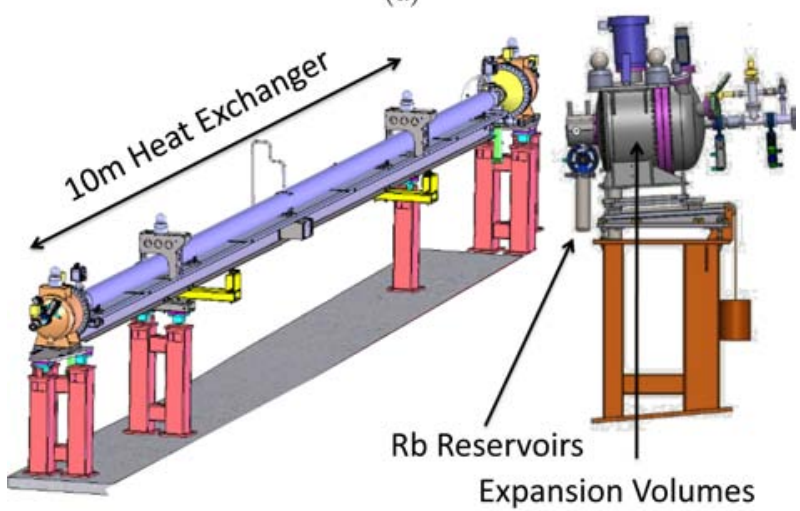

(b)

(c)

Fig. 11. (a) The principle of the vapor source: the Rb vapor flows from the reservoirs, and fills the inner tube, which is heated by a heat exchanger. (b) Technical drawing of the source, showing the Rb reservoirs (one at each end), the $10 \mathrm{~m}$ heat exchanger (outer tube), and the expansion volumes for the $\mathrm{Rb}$ vapor. (c) Detailed technical drawing of the source ends. Drawings courtesy of WDL Limited, UK and CERN.

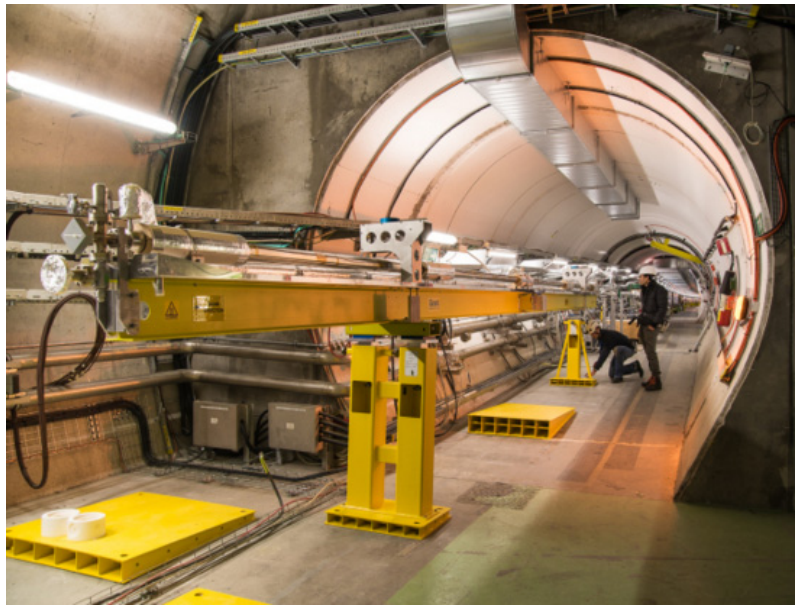

Fig. 12. Picture of the heat exchanger part of the vapor source as installed in the AWAKE facility. The view is looking up towards the SPS. Picture courtesy of CERN.

along the $10 \mathrm{~m}$ heat exchanger remains within $0.3^{\circ} \mathrm{C}$ around $220^{\circ} \mathrm{C}$ or $\sim 500 \mathrm{~K}$, satisfying the requirement $\delta T / T=\delta n_{\mathrm{Rb}} / n_{\mathrm{Rb}}<0.2 \%$ (or $1 \mathrm{~K}$ ). Rb ions also have a relatively large mass ( $\mathrm{Rb}$ atomic mass 85 or 87 ), 
which means that their large inertia can help mitigate the effects of ion motion [50].

Note that since the vapor and thus the plasma density are controlled through imposing temperatures on the system, one may envisage a vapor source with various temperatures corresponding to various densities. One could in particular realize a density step to stabilize the proton beam evolution mentioned in the previous section.

\subsubsection{Density ramp}

At the end of the $10 \mathrm{~m}$ heat exchanger, apertures allow the $\mathrm{Rb}$ vapor to expand into a large volume. Reservoirs at each end evaporate the $\mathrm{Rb}$ and (mostly) confine the vapor flow from the reservoir to the expansion volume, through the aperture. A theoretical model shows that the scale length for density variations along the system axis is on the order of the aperture diameter, which is $10 \mathrm{~mm}$, and direct simulation Monte Carlo (DSMC) results confirm the length of the density ramp $[59,66]$.

\subsubsection{Density gradient}

Symmetry between the two ends of the vapor source cannot be guaranteed by design, which leads to a density gradient along the source. This density gradient can be controlled or eliminated by independently controlling the $\mathrm{Rb}$ reservoir temperatures. Plasma density gradients can be used to compensate for dephasing of the accelerated particles and the wakefields and reach larger final energies [67]. The vapor density at each end of the source is measured using white light interferometry near the $D_{2}$ optical transition of $\mathrm{Rb}[68]$.

\subsubsection{Vapor ionization and SMI seeding}

In AWAKE the vapor ionization to create the plasma and the seeding of the SMI are achieved using a short $(\simeq 100 \mathrm{fs})$ and intense laser pulse. The laser system is a commercial chirped pulse amplification (CPA) system with an erbium: doped oscillator at $780 \mathrm{~nm}$ with a bandwidth of $\pm 5 \mathrm{~nm}$. The laser can produce up to $450 \mathrm{~mJ} /$ pulse at a repetition rate of $10 \mathrm{~Hz}$. It is focused to a radius of $1 \mathrm{~mm}$ near the vapor source entrance by an adjustable telescope. The Rayleigh length of the beam is $\sim 4 \mathrm{~m}$. Calculations [65] show that an energy on the order of $70 \mathrm{~mJ}$ is sufficient for ionization over the $10 \mathrm{~m}$ length and $1 \mathrm{~mm}$ minimum radius.

In order to seed the SMI, the laser pulse copropagates with the proton bunch and is placed within the proton bunch. [See (2), Fig. 9.] The abrupt plasma density creation (over a time much shorter than the plasma wave period, $\sim 1 / \omega_{\text {pe }}>3 \mathrm{ps}$ ) effectively seeds the SMI [69]. The laser pulse intensity is too low to drive significant wakefields and does not seed the instability.

The laser oscillator will also produce the seed for the laser that will drive the RF photoinjector gun, discussed below.

\subsection{SMI detection}

The first experiments will be dedicated to the study of the SMI of the proton bunch along the plasma. The occurrence of the SMI results in defocusing of some of the proton bunch particles, in charge modulation of the bunch along its length as well as in the generation of wakefields sustained by an electron plasma density modulation. All three effects can in principle be measured.

The first evidence of SMI will come from comparing the transverse images of the proton bunch after the vapor source, with and without plasma [70]. The occurrence of the SMI leads to the formation of a halo of the defocused proton.

Two diagnostics for the proton bunch modulation are based on transition radiation (TR). TR is emitted when the proton traverses the interface between two dielectric media (vacuum and metal in AWAKE).

The radiation is prompt and incoherent in the optical regime, where the radiation wavelength is much larger than the bunch's typical size or modulation. It is known as optical transition radiation (OTR). The OTR can be imaged onto the entrance slit of a streak camera with ps resolution. Therefore, the light modulation expected from the charge density modulation along the bunch axis can be directly observed and its frequency measured. For the electron plasma density range of AWAKE, the modulation frequency (approximately equal to the plasma frequency) lies in the $100-300 \mathrm{GHz}$ frequency range. It was shown experimentally that modulation frequencies in this range can be detected by the ps resolution streak camera [71]. The OTR light was mocked using two laser beams with beating frequencies in 
the above range and gated at the ns timescale (similar to the proton bunch duration). The modulation frequency is measured for the power spectrum of the Fourier transform of the streak camera image along the time axis. The start of the SMI development along the bunch can also be correlated with the location of the ionizing laser pulse to demonstrate the seeding effect. This is obtained by operating the streak camera in a slow mode with a ns timescale.

The TR is coherent for wavelengths longer than the bunch's typical size or modulation. It is then known as coherent transition radiation (CTR), and is often used to measure longitudinal bunch length. While the SMI leads to a radial charge modulation with longitudinal periodic modulation, calculations show that the CTR collected in a finite forward angle contains information about the SMI [72] and that it is emitted with significant power. This is because in AWAKE the peak longitudinal wakefield amplitude reaches a significant fraction of the wavebreaking value $\left(E_{z, \max } / E_{\mathrm{WB}} \sim 0.5\right)$, and thus the $p^{+}$bunch reaches full radial modulation, i.e. consists of truly longitudinally well-separated microbunches within the accelerating structure. In addition, the typical diverging angle of the defocused $p^{+}$is $\sim 1$ mrad and they are therefore at radii larger than $\lambda_{\text {pe }}$ at the diagnostic and do not radiate coherently at the modulation wavelength. The modulation frequency will be measured using microwave heterodyne measurement techniques. The CTR signal of unknown frequency is mixed in a nonlinear microwave diode with a signal of known frequency, chosen close to the plasma frequency expected from the plasma density. The frequency difference is then directly measured with a large bandwidth oscilloscope $(\sim 40 \mathrm{GHz})$.

\subsection{Electron injection}

Although the wakefield phase velocity is relatively small $\left(\gamma_{\text {phase }} \simeq \gamma_{\text {proton }}\right)$, the wakefield amplitude is also relatively small $(\sim 1 \mathrm{GV} / \mathrm{m})$ and trapping of plasma electrons is unlikely. External injection is therefore a priori necessary. The first injection experiments will use an electron bunch longer than the wakefield periods. This eliminates the need for timing synchronization between the electron bunch and the wakefields at the wakefield period scale. In this case, the plasma will trap and self-select a subset of the injected bunch particles. Simulation results
[59] indicate that GeV-energy beams with a narrow relative energy spread (a few \%) can be obtained. The fraction of electrons trapped is small.

Trapping of plasma electrons might be possible using a shorter, lower-emittance proton bunch, which may be available after the LHC LS2, and a corresponding higher plasma density.

During the growth phase of the SMI, along the first $\sim 4 \mathrm{~m}$ of plasma, the phase velocity of the wakefields varies from smaller than that of the proton bunch to superluminous [60]. This effect, similarly to the plasma density variation mentioned above, can lead to the loss of the externally injected particles. Various injection geometries have therefore been considered for AWAKE, side injection, "oblique" injection and on-axis injection.

\subsubsection{Side electron injection}

Injection at a location along the plasma after the SMI has saturated has been proposed [75]. However, this scheme is not practical, since the plasma is contained in a closed vacuum pipe $40 \mathrm{~mm}$ in diameter and no screen or measurement is located inside the $\mathrm{Rb}$ vapor. It also requires a dipole magnet around the plasma source to bend the electron beam toward the plasma, where it must cross the wakefields to have a chance to be trapped.

\subsubsection{Oblique electron injection}

Oblique electron injection consists in having the electron beam cross the axis of the proton beam a short distance $(\sim 1 \mathrm{~m})$ into the plasma [59]. The two beam vectors, and thus the crossing and injection point, can be determined using the two upstream screens. In this injection scheme, the electrons do not completely avoid the plasma region where the SMI grows. Figure 13 shows the electron energy spectrum expected with baseline experimental parameters and oblique injection.

\subsubsection{On-axis electron injection}

On-axis injection is the only geometry that can lead to low final emittance (assuming that emittance is otherwise preserved along the plasma). However, it is also the most sensitive to the wakefield evolution during the SMI growth, since electrons travel within the wakefields from the beginning of the plasma. 


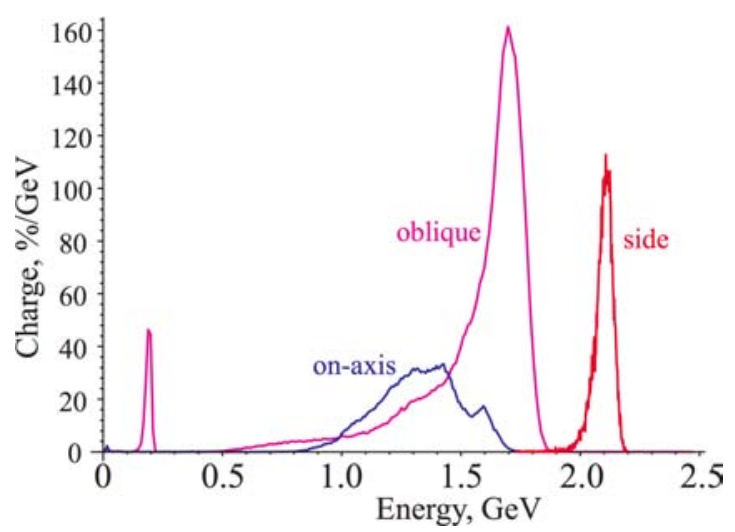

Fig. 13. The final simulated energy spectra of electrons in cases of the side, on-axis and oblique injection methods. (From Ref. 59.)

Simulation results predict small trapping efficiency in this geometry; however, it will be tested experimentally, due to its simplicity and potential for lower final emittance. Operation with two plasma sources may avoid this issue.

\subsubsection{Electron injector}

The electron injector is an S-band, 2.5-cell RF photoinjector gun developed by the CLIC project [73]. The unloaded accelerating gradient is $\sim 100 \mathrm{MV} / \mathrm{m}$ for a klystron power of $20 \mathrm{MW}$. The photocathode is driven by a laser system using the same oscillator as the ionizing laser, thereby insuring synchronization of the gun's RF oscillator and laser oscillator train at the sub-100 fs level. Synchronization between the ionizing laser pulse and the electron bunch is of sub-ps level. The gun is followed by a $2 \pi / 3 \mathrm{~S}$-band traveling accelerating structure powered by the same klystron as the gun. It delivers a $9.6 \mathrm{MV} / \mathrm{m}$ gradient for a $15 \mathrm{MW}$ input power. The final electron bunch energy is adjustable up to $20 \mathrm{MeV}$. The bunch charge is adjustable up to about $200 \mathrm{pC}$. The minimum normalized emittance is $2 \mathrm{~mm}$-mrad and the bunch length is typically $4 \mathrm{ps}$. The electron bunch is transported to the plasma, where it can be focused to a transverse size of $\sim 250 \mu \mathrm{m}$. The transport line can accommodate two of the injection schemes described above: oblique and on-axis injection. It has enough flexibility to place the beam waist at various locations over the first few meters of the plasma. Two screens placed in the common electron and proton beam line upstream of the plasma and equipped with various screens allow for overlap in space and time or for relative position and angle measurements between the beams. The beam line is well diagnosed with RF beam position monitors, pepper-pot emittance measurement, etc. A schematic of the integration of the RF gun in the AWAKE facility is shown in Fig. 14.

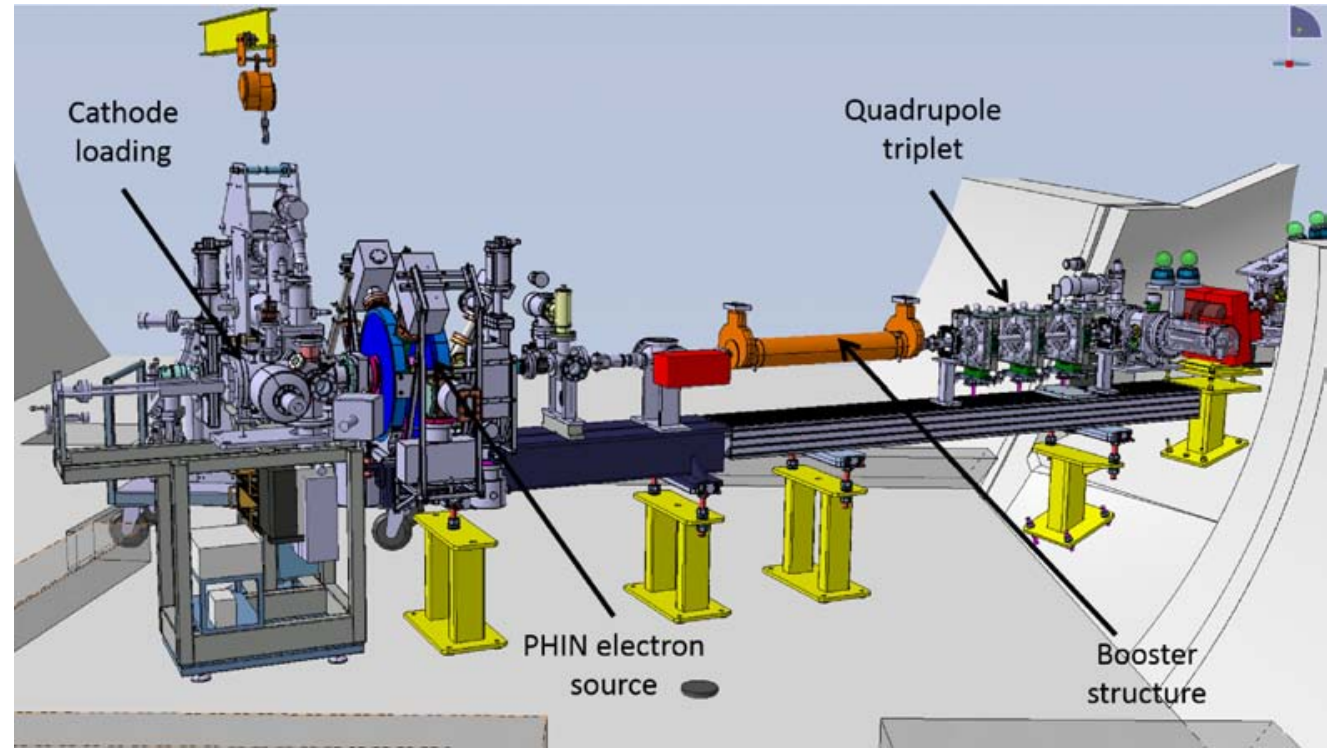

Fig. 14. Schematic of the RF gun (PHIN) integrated into the AWAKE facility (from Ref. 58). The electrons travel from the gun toward the proton beam line on the right-hand side through a dedicated tunnel. 


\subsubsection{Energy gain}

For AWAKE the initial beam-to-plasma-density ratio is around $6 \times 10^{-3}$, much less than unity. This means that the initial beam-plasma interaction is in the linear PWFA regime. After development of the SMI and where the electron injection should occur, approximately one proton bunch rms length behind the seeding laser pulse, the wakefields reach a fraction of the wave-breaking field [39]. The accelerating fields are in this case described by nonlinear theory $[4,5]$ and are strongly dependent on the longitudinal position, leading to a significant final energy spread for a finite length injected bunch.

The various electron injection geometries are expected to yield final energies in the $\mathrm{GeV}$ range with a few- $\%$ relative energy spread. The AWAKE electron spectrometer is based on a dipole magnet available at CERN [78]. The magnet provides a bending field with an integrated strength of $1.3-1.6 \mathrm{Tm}$. It is equipped with a wide, $\sim 1.5 \mathrm{~m}$ screen, so as to be able to observe a wide energy range (0.5$2.5 \mathrm{MeV}$ ) in a single event. It is also equipped with a quadrupole doublet, in order to image the beam at the plasma entrance onto the screen. With the doublet powered, the energy resolution reaches the sub$\%$ level over a narrow energy range. Focusing in the vertical (nondispersive) plane strongly increases the signal-to-background ratio. The background signal originates from the secondary particles produced by protons in upstream screens.

\subsubsection{Beam loading}

The energy spread of an accelerated electron bunch could potentially be improved by appropriate beam loading of the wakefield, a well-known method to decrease the energy spread in PWFA [74] as well as conventional accelerators. Beam loading may only be considered for the on-axis injection scheme, since the wakefields of the injected bunch are on the order of those of the drive bunch and thus significantly perturb them, potentially preventing trapping in the offaxis schemes. Initial numerical simulations consider the case of on-axis injection into a separate plasma cell located after the initial plasma cell in which the SMI develops [76]. Simulation results obtained with a premodulated, shorter test proton bunch indicated that beam loading indeed reduces the final energy spread [77]. The possibility of producing a short witness bunch that can properly load the wakefield, for an already-modulated driver, is one of the motivations for running with two plasma sources for Run 2 of AWAKE [76].

\subsection{Integration}

Integrating into an existing facility all the various systems necessary for AWAKE is a challenge. Figure 15 shows a schematic of the AWAKE facility with its main components. The proton bunch extracted from the SPS is focused at the entrance of the vapor cell. It travels through a horizontal chicane that allows for the injection of the ionizing laser pulse along its final trajectory, as shown in Fig. 9. The laser is hosted in a parallel tunnel transformed into a clean and temperature-controlled room. The laser beam is transported to the proton beam line, where light bleeding through a mirror is used for a "virtual plasma" analysis. Screens placed upstream

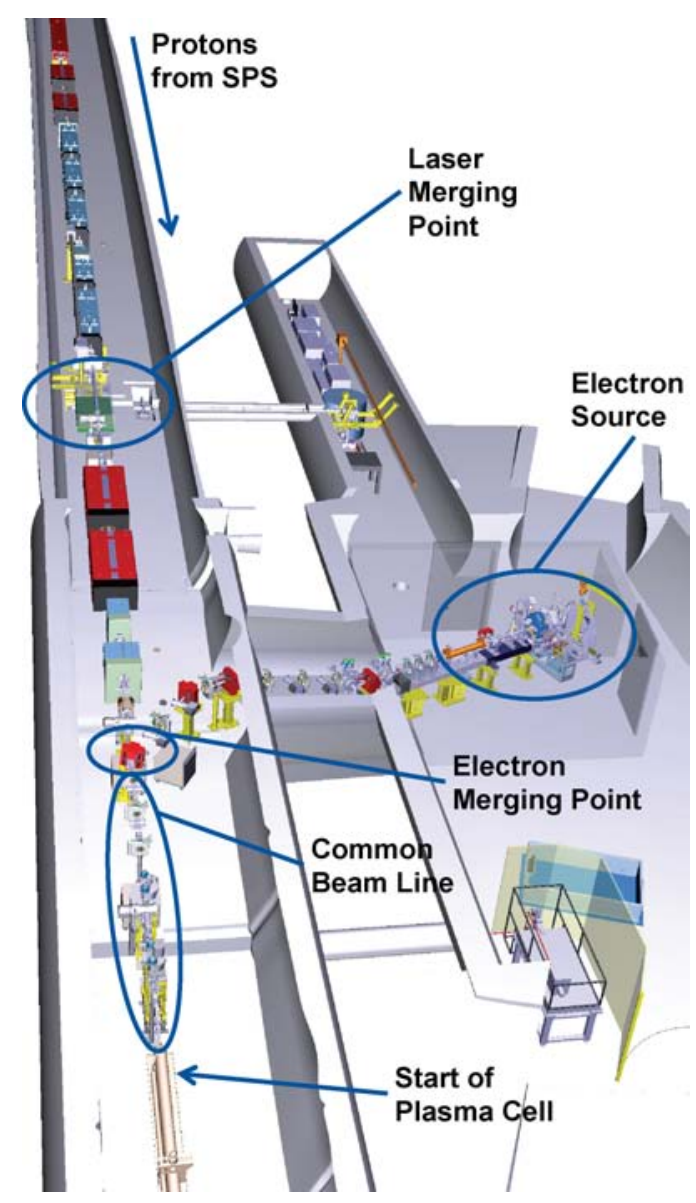

Fig. 15. Integration of the experimental apparatus in the AWAKE facility. (From Ref. 58.) 
and downstream of the plasma source allow spatial alignment of the proton and laser beams. One of the upstream screens is used to time the proton bunch and the laser beam using OTR and a streak camera. The proton beam diagnostics are located downstream of the plasma and consist of four screens. They are protected from the intense laser pulse by a light-tight laser beam dump. Two screens, separated by $\sim 8 \mathrm{~m}$, will be used to measure the details of the bunch transverse profile. Two will be used for OTR and CTR diagnostics.

The electron beam is transported toward the proton beam line, where its trajectory can be made collinear with, parallel to, or with an angle with respect to that of the proton beam. The light from the electron spectrometer screen is transported to another parallel tunnel where the CCD camera is located, shielded for the proton tunnel radiation.

\subsubsection{Synchronization}

On one hand and as discussed above, the laser pulse used for ionization that sets the initial phase of the wakefield and that will drive the electron gun photocathode are derived from a single laser oscillator and can be synchronized in a relatively standard way since these both use CW oscillator sources that can be phase-locked. On the other hand, the SPS is a "single-shot" machine that requires around $4 \mathrm{~s}$ to produce a single proton bunch and whose RF phase is constantly changed.

Therefore, once the SPS produces a $400 \mathrm{GeV}$ bunch for AWAKE, the bunch is stored for up to $500 \mathrm{~ms}$ to allow the phase-locking of the "transient" SPS RF at $\sim 200 \mathrm{MHz}$ to the laser modelocker frequency of $\sim 88 \mathrm{MHz}$. The long storage time is necessary to ensure that the SPS proton bunch can be extracted synchronously with the $10 \mathrm{~Hz}$ high-power laser pulse. Specific frequency multipliers and dividers had to be developed to lock the no-harmonic frequencies of the various systems. A phase-compensated fiber system was also developed to transmit and maintain the relative phase between AWAKE and the SPS RF over more than $3 \mathrm{~km}$.

\subsection{AWAKE: future steps}

The AWAKE experiment as described above is scheduled to run until the end of 2018, the time of the start of the LHC Long Shutdown 2. Experience has already shown that the development and integration of an experiment of the scale of AWAKE takes years. Work has therefore started toward exploring and defining experiments and studies that will take place after this shutdown.

The most relevant topics are:

- The production of a high-quality (low emittance and energy spread), high-energy (>1 GeV) electron bunch;

- The development of plasma sources scalable to very long lengths;

- Short and long-term applications of the acceleration scheme.

These are briefly discussed below.

\subsubsection{Witness bunch acceleration}

Production of a high-quality particle bunch is one of the holy grails of plasma-based particle accelerators. It seems clear that when one is using the SMI of the drive bunch to reach large wakefield amplitudes and for reasons described above, it is necessary to separate the self-modulation plasma from the acceleration plasma.

The separation and operation of two independent plasma sources allows, in principle, on-axis injection of external particles after the SMI has evolved. However, this also brings a number of challenges that were avoided in the first phase of AWAKE.

The self-modulated proton bunch evolves once it exits the plasma and enters another. The beam and plasma therefore have to find a new equilibrium between their modulated densities. This leads to some evolution of the wakefields in phase and amplitude at the entrance of the second source, which also has its own density ramp.

Since the beam and plasma form a resonant system, the plasma density between the two sources must be adjusted (and reproducible) at the (sub-)\% level.

The optimum witness bunch parameters must be determined. The advantage of operation at relatively low plasma density (compared to other plasmabased accelerators) is that the size of the accelerating structure is relatively large $(\mathrm{mm})$. The size of the structure determines the size of the witness bunch as well as the tolerances in terms of spatial 
and temporal alignment. For example, the larger structure size means that reasonably long bunches (tens of microns) can be used, possibly benefiting from beam loading.

Alternatively, the very-short, very-high-peakcurrent bunches typically produced by LWFA could be considered for injection into the accelerating structure and have advantageous properties [79]: high-current and short compared to the accelerating structure, intrinsic synchronization (derived from the same laser), high energy of injection, etc. The source itself is also more compact than an RF-based one.

\subsubsection{Development of scalable plasma sources}

The current AWAKE plasma source meets many of the requirements, such as density uniformity, a short entrance ramp, stability and reproducibility, seeding of the SMI, or the possibility of producing a density step. However, it uses one TW-class laser beam for every $10-20 \mathrm{~m}$, which for long plasmas would be costly and would require staging of the plasma in order to inject fresh laser beams. Alternative plasma source technologies, with potentially better scalability, are therefore being investigated.

\subsubsection{Helicon source}

Plasma can be created by nonresonant coupling of an RF wave (e.g. industry $13.6 \mathrm{MHz}$ ) to a weakly magnetized plasma in a helicon source. A helicon unit cell plasma source may thus consist of two coils to produce a uniform longitudinal magnetic field and of an $\mathrm{RF}$ antenna wrapped around the vacuum chamber. Helicon sources are thus interesting because they are modular, with only one entrance and one exit for the proton and electron bunches. These sources usually operate at densities two orders of magnitude lower than densities relevant to AWAKE. However, it was recently demonstrated at the Institute for Plasma Physics-Greifswald that a density of $7 \times 10^{14} \mathrm{~cm}^{-3}$ can be reached. The issues of density uniformity and reproducibility have to be addressed and will be the focus of a new effort between the Institute for Plasma Physics-Greifswald, the Swiss Plasma Center-Lausanne, CERN and AWAKE.

\subsubsection{Discharge source}

Electrical discharges between two electrodes separated by long distances $(\sim 10 \mathrm{~m})$ have already produced densities relevant to AWAKE. They are much simpler and more affordable devices than a TW-class laser. It is, however, not clear whether the density uniformity and reproducibility are commensurate with the SMI requirements. The issue of space between discharges of finite length must also be resolved.

\subsection{Toward applications}

While AWAKE is in its final construction phase at the time of writing, for experiments on SMI studies in late 2016 and electron external injection studies in 2017-18, AWAKE is preparing Run 2 [76], scheduled for after the LHC LS2 (2021). This preparation includes a first scientific program that could be realized within the current facility. Possible themes include: operation with shorter, lower-emittance proton bunches in higher-density plasmas; increasing the accelerating gradient; operation with two plasma sources (for SMI and acceleration); external injection of a short electron bunch in the second plasma source; and acceleration of an electron bunch with narrow energy spread and low emittance, as discussed above. Acceleration of positron bunches is also envisaged.

At the same time, mid- and long-term plans are also considered. An appealing path toward PDPWFA applications is to use the existing CERN proton beams not only as plasma drivers, but also for HEP applications in the form of a high-energy electron-proton (eP) collider fitting on the CERN site. A basic concept for a PD-PWFA-based eP collider has been studied in Ref. 80. The principle is shown in Fig. 16. An LHC bunch could potentially drive electrons up to $\mathrm{TeV}$ energies [36], while an SPS bunch could potentially drive electron energies similar to those of the proposed LHeC collider (electron energies of $60-100 \mathrm{GeV}$ ) [81]. A PD-PWFA as proposed in Ref. 80 would have a several-orders-ofmagnitude lower luminosity compared to the LHeC proposal [81], due to the relatively low repetition rate of the available proton beams used to drive the wakefields. However, a significant advantage of targeting eP applications is that the requirements for electron beam emittance are orders of magnitude more relaxed than for electron-positron linear colliders [81-83], imposing less demanding needs for emittance preservation during the plasma acceleration. Since the AWAKE experiment uses the SPS beam for the proof-of-principle experiments, the path 


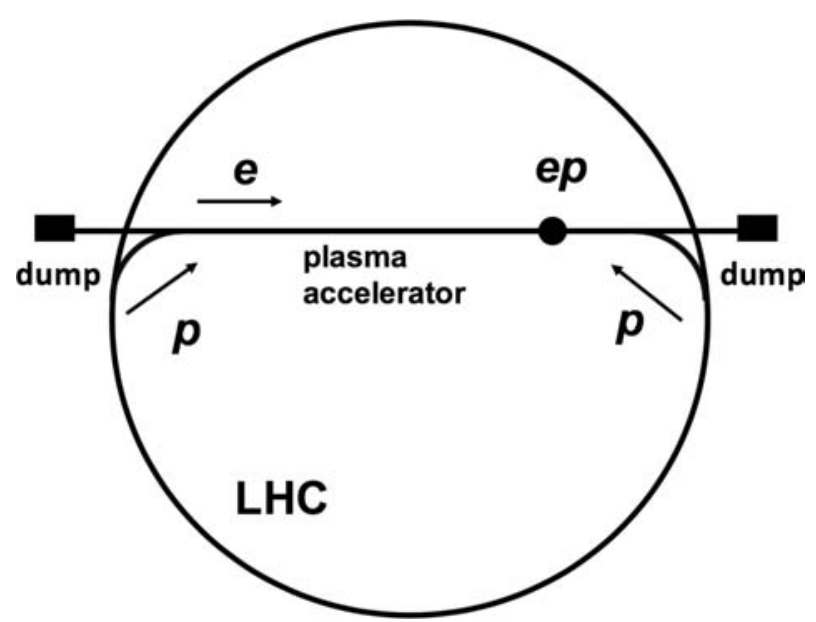

Fig. 16. A basic concept for a PD-PWFA-based electronproton collider. One LHC bunch is used to accelerate an electron bunch to $\mathrm{TeV}$ energies before the electron bunch collides with a second LHC bunch. This type of application maximizes the use of existing proton accelerator infrastructure at CERN. (From Ref. 80.)

from AWAKE experiments to eP collisions with LHeC-energy bunches could potentially be reasonably short, without a need for large intermediate test facilities.

\section{Acknowledgments}

We gratefully acknowledge useful discussions with Konstantin Lotov concerning the self-modulation instability as well as AWAKE simulations in general, with Allen Caldwell about the early phases of AWAKE, and with Vladimir Shiltsev for information about PROTOPLASMA. Furthermore, we thank Ioanis Kourbanis, Sergey Ivanov and Susumu Igarashi for providing the main parameters for the Fermilab Main Injector, IHEP U70 and J-PARC synchrotrons, respectively.

\section{References}

[1] T. Tajima and J. M. Dawson, Laser electron accelerator, Phys. Rev. Lett. 43, 267 (1979).

[2] Laser-driven plasma acceleration, in $R A S T$, this volume (2016).

[3] P. Chen, J. Dawson, R. Huff and T. Katsouleas, Acceleration of electrons by the interaction of a bunched electron beam with a plasma, Phys. Rev. Lett. 54, 693 (1985).

[4] Electron-beam-driven plasma acceleration, in RAST, this volume (2016).

[5] W. Lu, C. Huang, M. M. Zhou, W. B. Mori and T. Katsouleas, Phys. Plas. 12, 063101 (2005).
[6] B. Allen, V. Yakimenko, M. Babzien, M. Fedurin, K. Kusche and P. Muggli, Experimental study of current filamentation instability, Phys. Rev. Lett. 109, 185007 (2012).

[7] M. Zhou et al., Beam head erosion in self-ionized plasma wakefield accelerators, in Proc. PACO7 (2007), p. 3064.

[8] C. A. Lindstrøm et al., Staging optics considerations for a plasma wakefield acceleration linear collider, Nucl. Instrum. Methods in Phys. Res. A 829, 224 (2016).

[9] W. Lu et al., High transformer ratio PWFA for application on XFELs, in Proc. PAC09 (2009), p. 3028.

[10] E. Kallos et al., Plasma wakefield acceleration utilizing multiple electron bunches, in Proc. PACO7 (1997), p. 3070.

[11] I. Blumenfeld et al., Scaling of the longitudinal electric field and transformer ratio in a nonlinear plasma wakefield accelerator, Phys. Rev. ST Accel. Beams 10, 111301 (2010).

[12] B. E. Blue, Plasma wakefield acceleration of an intense positron beam, Ph.D. thesis (UCLA, 2003).

[13] A. Caldwell, K. Lotov, A. Pukhov and F. Simon, Proton-driven plasma-wakefield acceleration, Nat. Phys. 5, 363 (2009).

[14] S. Lee, T. Katsouleas, R. G. Hemker, E. S. Dodd and W. B. Mori, Plasma-wakefield acceleration of a positron beam, Phys. Rev. E 64, $045501 R$ (2011).

[15] K. V. Lotov, Simulation of proton-driven plasma wakefield acceleration, Phys. Rev. ST Accel. Beams 13, 041301 (2010).

[16] R. Assmann et al., Generation of short proton bunches in the Cern Accelerator Complex, in Proc. PAC09 (2009), p. 4542.

[17] S. Nagaitsev, Fermilab Antiproton Source, Recycler Ring, and Main Injector. FERMILAB-FN-0957-AD (2013).

[18] T. Koseki et al., Beam commissioning and operation of the J-PARC main ring synchrotron, Prog. Theor. Exp. Phys. 02, B004 (2012).

[19] S. Ivanov, Accelerator complex U70 of IHEP: status and upgrades, in Proc. RuPAC2014 (2014), TUX02.

[20] J. M. Dawson, Nonlinear electron oscillations in a cold plasma, Phys. Rev. 113, 383 (1959).

[21] A. Caldwell et al., Preliminary study of proton driven plasma wakefield acceleration, in Proc. PAC09 (2009), p. 4551.

[22] G. Xia and A. Caldwell, Producing short proton bunch for driving plasma wakefield acceleration, in Proc. IPAC10 (2010), p. 4395.

[23] P. Schutt, T. Weiland and V. M. Tsakanov, On the wakefield acceleration using the sequence of driving bunches. DESY M-88-13 (1988), p. 12.

[24] D. H. Whittum, Transverse two-stream instability of a beam with a Bennett profile, Phys. Plas. 4, 1154 (1997). 
[25] K. Lotov, Instability of long driving beams in plasma wakefield accelerators, in Proc. EPAC08 (1998), p. 806.

[26] E. Esarey, J. Krall and P. Sprangle, Envelope analysis of intense laser pulse self-modulation in plasmas, Phys. Rev. Lett. 72, 2887 (1994).

[27] W. B. Mori, The physics of the nonlinear optics of plasmas at relativistic intensities for shortpulse lasers, IEEE Trans. Plas. Sci. 33, 1942 (1997).

[28] N. Kumar, A. Pukhov and K. Lotov, Selfmodulation instability of a long proton bunch in plasmas, Phys. Rev. Lett. 104, 255003 (2010).

[29] A. Pukhov et al., Phase velocity and particle injection in a self-modulated proton-driven plasma wakefield accelerator, Phys. Rev. Lett. 107, 145003 (2011).

[30] J. Vieira, P. Muggli and W. B. Mori, Hosing instability in self-modulated plasma wakefields, Phys. Rev. Lett. 112, 205001 (2014).

[31] E. Adli et al., Letter of intent for a demonstration experiment in proton-driven plasma wakefield acceleration. CERN-SPSC-2011-020 (2011).

[32] J. C. T. Thangaraj et al., PROTOPLASMA proton-driven plasma-wakefield experiment at Fermilab: stages and approach. FERMILAB-CONF12-409-APC (2012).

[33] K. V. Lotov, Controlled self-modulation of high energy beams in a plasma, Phys. Plas. 18, 024501 (2011).

[34] K. V. Lotov, Effect of beam emittance on selfmodulation of long beams in plasma wakefield accelerators, Phys. Plas. 22, 123107 (2015).

[35] K. V. Lotov, Physics of beam self-modulation in plasma wakefield accelerators, Phys. Plas. 22, 103110 (2015).

[36] A. Caldwell and K. Lotov, Plasma wakefield acceleration with a modulated proton bunch, Phys. Plas. 5, 103101 (2011).

[37] K. V. Lotov, Simulation of ultrarelativistic beam dynamics in plasma wakefield accelerator, Phys. Plas. 5, 785 (1998).

[38] K. V. Lotov, Fine wakefield structure in the blowout regime of plasma wakefield accelerators, Phys. Rev. ST Accel. Beams 6, 061301 (2003).

[39] K. V. Lotov, Excitation of two-dimensional plasma wakefields by trains of equidistant particle bunches, Phys. Plas. 20, 083119 (2013).

[40] P. Piot et al., Generation and characterization of electron bunches with ramped current profiles in a dual-frequency superconducting linear accelerator, Phys. Rev. Lett. 108, 034801 (2012).

[41] M. Boscoloa, M. Ferrario, I. Boscolob, F. Castellib and S. Cialdi, Generation of short $\mathrm{THz}$ bunch trains in a RF photoinjector, Nucl. Instrum. Methods in Phys. Res. A 577, 409 (2007).

[42] P. Muggli, V. Yakimenko, M. Babzien, E. Kallos and K. P. Kusche, Generation of trains of electron microbunches with adjustable subpicosecond spacing, Phys. Rev. Lett. 101, 054801 (2008).

[43] Y.-E. Sun et al., Tunable subpicosecond electronbunch-train generation using a transverse-tolongitudinal phase-space exchange technique, Phys. Rev. Lett. 105, 234801 (2010).

[44] Y. Fang, W. Mori and P. Muggli, Numerical study of self-modulation instability of $1 \mathrm{nC}$ electron bunch at ATF, AIP Conf. Proc. 1507, 559 (2012).

[45] J. Vieira, Y. Fang, W. B. Mori, L. O. Silva and P. Muggli, Transverse self-modulation of ultrarelativistic lepton beams in the plasma wakefield accelerator, Phys. Plas. 19, 063105 (2012).

[46] Y. Fang et al., Seeding of self-modulation instability of a long electron bunch in a plasma, Phys. Rev. Lett. 112, 045001 (2014).

[47] Facility for Advanced Accelerator Experimental Tests at SLAC. http://facet.slac.stanford.edu.

[48] E. Adli et al., Progress of plasma wakefield selfmodulation experiments at FACET, Nucl. Instrum. Methods in Phys. Res. A 829, 334 (2016).

[49] O. Lishilin et al., First results of the plasma wakefield acceleration experiment at PITZ, Nucl. Instrum. Methods in Phys. Res. A 829, 37 (2016).

[50] J. Vieira, R. A. Fonseca, W. B. Mori and L. O. Silva, Ion motion in self-modulated plasma wakefield accelerators, Phys. Rev. Lett. 109, 145005 (2012).

[51] J. Vieira, R. A. Fonseca, W. B. Mori and L. O. Silva, Ion motion in the wake driven by long particle bunches in plasmas, Phys. Plas. 21, 056705 (2014).

[52] Y. Fang, J. Vieira, L. D. Amorim, W. Mori and P. Muggli, The effect of plasma radius and profile on the development of self-modulation instability of electron bunches, Phys. Plas. 21, 056703 (2014).

[53] L. Yi et al., Scheme for proton-driven plasmawakefield acceleration of positively charged particles in a hollow plasma channel, Phys. Rev. ST Accel. Beams 16, 071301 (2013).

[54] L. Yi et al., Scheme for proton-driven plasmawakefield acceleration of positively charged particles in a hollow plasma channel, Phys. Rev. ST Accel. Beams 16, 071301 (2013).

[55] L. Q. Yi et al., Positron acceleration in a hollow plasma channel up to TeV regime, Sci. Rep. 4, 4171 (2014).

[56] M. Chung et al., Studies of the self-modulation and other instabilities in proton beam-driven plasma wakefield accelerators. URSI Asia-Pacific Radio Science Conference, Seoul, South Korea (2016).

[57] R. Assmann et al., Proton-driven plasma wakefield acceleration: a path to the future of high-energy particle physics, Plas. Phys. Control. Fus. 56, 084013 (2014).

[58] E. Gschwendtner et al., AWAKE, the advanced proton driven plasma wakefield acceleration experiment at CERN, Nucl. Instrum. Methods in Phys. Res. A 829, 76 (2016). 
[59] A. Caldwell et al., Path to AWAKE: evolution of the concept, Nucl. Instrum. Methods in Phys. Res. A 829, 3 (2016).

[60] K. V. Lotov et al., Electron trapping and acceleration by the plasma wakefield of a self-modulating proton beam, Phys. Plas. 21, 123116 (2014).

[61] K. V. Lotov et al., Parameter sensitivity of plasma wakefields driven by self-modulating proton beams, Phys. Plas. 21, 083107 (2014).

[62] K. Lotov et al., Effect of plasma inhomogeneity on plasma wakefield acceleration driven by long bunches, Phys. Plas. 20, 013102 (2013).

[63] C. B. Schroeder et al., Growth and phase velocity of self-modulated beam-driven plasma waves, Phys. Rev. Lett. 107, 145002 (2011).

[64] K. V. Lotov, Controlled self-modulation of high energy beams in a plasma, Phys. Plas. 18, 024511 (2011).

[65] E. Oz and P. Muggli, A novel Rb vapor plasma source for plasma wakefield accelerators, Nucl. Instrum. Methods in Phys. Res. A 740(11), 197 (2014).

[66] G. Plyushchev and R. Kersevan, to be published.

[67] A. Petrenko, K. Lotov and A. Sosedkin, Numerical studies of electron acceleration behind selfmodulating proton beam in plasma with a density gradient, Nucl. Instrum. Methods in Phys. Res. A 829, 63 (2016).

[68] E. Oz et al., An acccurate Rb density measurement method for a plasma wakefield accelerator experiment using a novel $\mathrm{Rb}$ reservoir, Nucl. Instrum. Methods in Phys. Res. A 829, 321 (2016).

[69] D. F. Gordon et al., Seeding of the forward Raman instability by ionization fronts and Raman backscatter, Phys. Rev. E 64, 046404 (2001).

[70] M. Turner et al., Indirect self-modulation instability measurement concept for the AWAKE proton beam,
Nucl. Instrum. Methods in Phys. Res. A 829, 314 (2016).

[71] K. Rieger, Master's thesis (Technical University of Munich, 2015).

[72] M. Martyanov, to be published.

[73] G. Bienvenu, CTF3 $3 \mathrm{GHz}$ RF gun test at CERN. CTF3-Note-093 (2009).

[74] M. Tzoufras et al., Beam loading in the nonlinear regime of plasma-based acceleration, Phys. Rev. Lett. 101, 145002 (2008).

[75] K. V. Lotov, Optimum angle for side injection of electrons into linear plasma wakefields, J. Plas. Phys. 78(4), 455 (2012).

[76] E. Adli, Towards Awake applications: electron beam acceleration in a proton driven plasma wake, in Proc. IPAC'16 (Busan, South Korea, 2016), p. 2557.

[77] V. K. Berglyd Olsen et al., Loading of a plasmawakefield accelerator section driven by a selfmodulated proton bunch, in Proc. IPAC'15 (Richmond, VA, USA, 2015), p. 2551.

[78] L. Deacon et al., A spectrometer for proton driven plasma accelerated electrons at Awake - recent developments, in Proc. IPAC'16 (Busan, South Korea, 2016), p. 2605.

[79] P. Muggli et al., Injection of a LWFA electron bunch in a PWFA driven by a self-modulated proton bunch, in Proc. IPAC'14 (Dresden, Germany, 2014), p. 1470.

[80] A. Caldwell and M. Wing, VHEeP: a very high energy electron-proton collider. arXiv: 1606.00783 [hep-ex].

[81] LHeC Study Group, CERN-OPEN-2012-01 (2012).

[82] CLIC Conceptual Design Report (2012).

[83] International Linear Collider Technical Design Report (2013).
Erik Adli did his Ph.D. research on linear colliders at CERN and his postdoctoral work on PWFA experiments at FACET/SLAC. He is currently an associate professor at the University of Oslo, Norway, where he is leading the Norwegian accelerator activities toward the AWAKE and CLIC projects at CERN, and toward ESS.
Patric Muggli is leading the Future Accelerators group at the Max Planck Institute for Physics in Munich, Germany. The main focus of his current research is the AWAKE project at CERN. He is a fellow of the American Physical Society and of IEEE. He received the IEEE Particle Accelerator Science and Technology Award in 2011. 